\title{
Congestive Heart Failure Management in a Home-Care System through the CHS Contact Center
}

\author{
N Maglaveras ${ }^{1}$, I Lekka ${ }^{1}$, I Chouvarda $^{1}$, D Adamidis ${ }^{2}$, H Karvounis $^{2}$, G Louridas $^{2}$, B Zeevi ${ }^{3}$, EA Balas $^{4}$ \\ ${ }^{1}$ Lab of Medical Informatics, ${ }^{2}$ A' AHEPA Cardiology Clinic, Aristotle University, Thessaloniki, \\ Macedonia, Greece, ${ }^{3}$ Card Guard, Scientific Survival, Rehovot, Israel, ${ }^{4}$ School of Public Health St. \\ Louis University, St. Louis, MO, USA
}

\begin{abstract}
The Citizen Health System (CHS) is a European Commission (CEC) funded project in the field of IST for Health. It has developed a generic contact center, which can be used in the monitoring, treatment and management of chronically ill patients at home such as congestive heart failure $(\mathrm{CHF})$ patients. The virtue of the CHS contact center is that using any type of communication and telematics technology it is able to provide timely and preventive prompting to the CHF patients, thus, achieving better disease management. In this paper, we present the quantitative and qualitative results throug the use of the CHS contact center by CHF patients, looking at the prevention of complications, the quality of life of the involved patients, and the quality of the medical procedures and interventions used for the CHF disease management. It is shown through specific evidence that the use of the monitoring and interventions through the CHS contact center prevents worsening of the patients condition, something evidenced and supported basically by the sharp reduction in hospitalisation rates for the CHF patients that used the CHS home-care system.
\end{abstract}

\section{Introduction}

Information technology (IT) applications in medicine are proven pivotal in the success of preventive medicine [1]. Today, due to the fast growing and penetration of the INTERNET and mobile telephone technology, the IT applications in the health care environment are focused at e-consultation [2] and home care delivery [3] and the use of triage systems [4]. Home care delivery is becoming a very important issue, especially for the management of chronic diseases such as diabetes, congestive heart failure, coronary heart disease, etc. IT based applications for home care delivery, are important media to increase health care quality, increase quality of life, and create a better educational platform with carefully designed and customisable patient prompting which in turn is expected to be instrumental in increasing the collaboration degree between the patient and the physician. However, in the case of heart failure there are ambiguous results regarding the effectiveness of IT technologies when used for telemonitoring and home care delivery [5-7].

Pivotal to these purposes are contact centers, which act as mediators between the medical staff and the citizens seeking advice and/or therapy. In this work, a generic contact center developed within the CHS project is used for the management and treatment of CHF patients [8]. Heart failure is a clinical syndrome that has become more prevalent in recent years. In 1996, almost 4.8 million Americans were afflicted with $\mathrm{CHF}$, and each year approximately 400000 new cases of CHF were diagnosed. Disease prevalence is expected to reach 10 million cases in the U.S. alone by the year 2007.

Heart failure is one of the main challenges of the physician as indicated by a high readmission rate, which has ranged from $29 \%$ to $47 \%$ within 3 to 6 months of the initial discharge. Behavioural factors, such as noncompliance with diet and medications, social factors, such as social isolation, and lack of education about the disease frequently contribute to early readmissions. These readmissions are a particularly appropriate target for intervention, because the population at risk is identified and the factors that contribute to this phenomenon are well known.

The aim of the study is to test the hypothesis that intensified treatment monitoring and guidance for patients with congestive heart failure through the use of telematic systems will lead to reduced CHF-related hospitalisation 
and mortality. It is shown that the use of the CHS contact center renders a more effective schema in the management of CHF patients and it has dramatic effects in the quality of health care delivery, the quality of life and the health delivery system since it favours procedures enabling preventive actions.

\section{Methods}

The trial was conducted from August 2001 through April 2003 at the Department of Cardiology of Aristotle University (AHEPA Hospital of Thessaloniki, Greece).

All study patients were recruited of hospitalised patients or other patients who had been hospitalised recently with known diagnosis of CHF irrespective of prior hospitalisations or outpatients of the heart failure clinic in the Department of Cardiology of Aristotle University (AHEPA Hospital of Thessaloniki). All the examinations and the laboratory tests took place in the AHEPA Hospital of Thessaloniki. All patients lived in the Thessaloniki district.

Upon recruitment to the study, patients received a consent form and instruction brochure for the contact centre and for the measurement devices. They also received their treatment plan that shows the schedule of their monitoring activities. Patients are asked how they prefer to communicate with the CHS contact centre (regular phone via the Automated Contact Centre, Web or WAP). Patients, who preferred WAP, were offered a WAP cellular phone and a special instruction brochure. Two out of the 15 patients received a WAP phone.

After signing the consent form, the clinician explains to the patient the CHS home monitoring procedure. The patient receives a digital scale, a digital blood pressure meter and a single lead ECG transtelephonic microdevice (CARDGUARD 2206).

The patients called in to report their measurements three times per week. The measurements are body weight, body temperature, heart rate, blood pressure and answers (yes or no) on five simple questions. The questions are: Were you breathless during the night? Are your legs swollen? Do you feel more tired today? Do you have more dyspnea today? Have you taken all the medications? Thus, the selected questions targeted two objective symptoms (paroxysmal nocturnal dyspnea and peripheral oedema), two subjective symptoms (fatigue and dyspnea) and a gentle reminder to take their medicine. Patients send single lead ECG signal via telephone once a week.

After patients concluded their session, they received a CHF education tip. The patient then had the opportunity to listen to one of 24 educational messages. Each educational message was approximately 12 minutes in length. Patients may call any time to receive information on the following topics about heart failure: Knowledge and Prevention, Treatment, Exercise, Salt, Fat, Cholesterol and Weight, Fluids and Alcohol.

Daily review of patient data was performed by a clinician at the Contact Centre. The cardiologist was contacted for advice in special cases during the week. The cardiologist reviewed patient data through the CHS Web application (Fig. 1). When needed he/she communicated with the patients either via SMS or through the telephone. The most common clinician interventions were "change in medication dose" (e.g. increase in dose of diuretics) and "advice for visit to the outpatient department".

\section{Results}

\subsection{Clinical assessment}

According to the scenario presented above, the patient can send data from virt ually everywhere, provided he/she has the portable devices necessary for the measurements of basic parameters such as arterial blood pressure, blood glucose etc. These recording devices are getting smaller and smaller, and many of them are supporting transtelephonic transmission, which may decrease the session duration, and reduce the number of errors. This technology together with prompting physicians can increase the effectiveness in preventive care in the future [9].

\begin{tabular}{|l|c|c|}
\hline \multicolumn{3}{|c|}{ All Causes } \\
\hline Prior Period & 14 & Hospital Days \\
\hline During CHS & 4 & 60 \\
\hline \multicolumn{3}{|c|}{ Cardiovascular Causes } \\
\hline Prior Period & 13 & 53 \\
\hline During CHS & 4 & 18 \\
\hline
\end{tabular}

Table 1. Hospitalisation rates before and during monitoring period using the CHS contact center.

For the CHF patients who participated in the study, the average follow up period was 10 months. Hospitalisation data are presented in Table 1. The number of hospitalisations per patient in the follow up period was 0,33 , compared with 1,16 per patient during the period before enrolment. Hospitalisation days declined from 5 per patient to 1,5 (in the follow up period). Table 2, 
shows hospitalisations for subgroups of patients, according to NYHA class. Patients in NYHA class II had fewer hospitalisations in the preceding period and less of reduction than class III patients during the monitoring period.

During the study, there were 32 physician notifications for 8 problems. The most common reported problem was weight gain, and dyspnea. Other problems were hypertension, hypotension and brady - or tachycardia.

\begin{tabular}{|l|cc|}
\hline & \multicolumn{1}{|l|}{ Admissions } & Hospital Days \\
\hline \multicolumn{3}{|c|}{ NYHA Class II } \\
\hline Prior Period & 6 & 23 \\
\hline During CHS & 2 & 7 \\
\hline \multicolumn{3}{|c|}{ NYHA Class III } \\
\hline Prior Period & 8 & 37 \\
\hline During CHS & 2 & 11 \\
\hline
\end{tabular}

Table 2. Hospitalisation rates presented according to the NYHA Class of the CHF patients.

\subsection{System assessment}

Assessment on system usage was based on Log File analysis and user satisfaction short questionnaire. All interaction via the Contact Centre has been recorded in $\log$ files. For each call a separate text $\log$ file was created with all the actions reported. The Log File Analyser was then used to evaluate the Contact Centre usage.

The average duration of a successful call is 147 seconds, i.e. less than two minutes. Compared to the average call for an obese/diabetes patient which is another important chronic group of patients this is considerably higher, since the heart failure patients sends more values and answers more questions.

Compliance was very high among the heart failure patients. From the 1574 expected contacts, 1204 contacts were made, i.e. $76 \%$.

Due to the limited number of enrolled patients, instead of a questionnaire, a set of questions were asked by phone in order to assess their satisfaction regarding system usage.

Their answers showed that patient acceptance of the program was high; all patients were satisfied in overall with the system and would recommend it. More than $80 \%$ would like to continue using it. All patients found the training they received very satisfactory. There were however, problems in contacting the contact centre for some patients. There are a number of reasons for that. There were frequent telephone line interruptions for patients living in a certain district. Additionally, there was only one line dedicated to other types of chronic patients so for a certain time period 80 patients in total were using the call centre. During the morning, where most people used to call, there were instances where the line was busy. $75 \%$ of the patients believed that the use of the system has improved their health status. This is also verified by the significant drop in hospitalisations during this period.

The clinicians involved in the trial were overall very satisfied with the system. Their comments led to many improvements on the clinician applications. Specifically, the addition of the Clinician Alert Tool was proven to be a timesaving mechanism for the nurse, since it filtered out the trivial contacts and indicated the significant ones, i.e. patient measurements out-of-range or schedule not followed, as soon as they were made. The cardiologist found the Clinician Web Interface simple and easy to use.

In general we can say that Home Care is facilitated since multiple means of communication are offered, and thus different patient groups are not excluded. This increases patients' involvement in their own health, and reduces hospitalization.

\section{Conclusion}

This paper describes a home care contact center able of extending beyond data collection by generating feedback and supporting patient education. It combines initial screening, use of measurement micro-devices, patient education, decision support, appropriate telephone/WAP/WEB contacts and physician access.

This system is currently tested in a congestive heart failure clinical trial emphasizing the management of $\mathrm{CHF}$ patients. Such trials will help assess user acceptance of the system and its clinical effectiveness.

An important limitation of the study is the low number of the patients that participated in the trial; this is due to the following reasons:

- CHF outpatient clinic is a new clinic with no preexisting pool of patients,

- patients with other severe chronic diseases (cancer, severe COPD, etc.) were excluded,

- patients NYHA class I or IV were excluded We excluded the patients NYHA I because the multidisciplinary disease management in these patient increase the total cost and the patients NYHA IV because their management is complicated, and

- due to the advanced age of the specific target group and their concerns with new technologies many patients were unwilling to participate. 
Since the number of patients is low, the hospitalisation rate was not compared to a control group but to the same patients' hospitalisations in the 10-month period prior to the trial. Based on our results hospitalisation rate dropped significantly for the 15 patients. The reason for this drop is the timely clinician intervention when significant changes in the vital signs and symptoms were reported. Since hospitalisation contributes over $60 \%$ of the total cost of heart failure to the health service, it is no surprising that considerable efforts have been expended in developing programs that reduce the risk of hospitalisation in patients with heart failure.

As in previous studies, our results indicate that use of a program that includes patient education, attention to medication compliance and monitoring of clinical and fluid status can substantially reduce hospitalisations. Compared with the same period of time before enrolment in the trial, hospitalisations were reduced more than $60 \%$. The reduction in admissions and hospital days was evident among the patients with more severe heart failure (Table 2). Patients were very satisfied from the "close follow-up" and that is verified both from the questionnaire and their compliance in using the system $(76 \%)$. It was noticed that in cases of older patients who were intimidated with technology other family members helped with the data transmission.

In summary, our findings suggest that telemonitoring with CHS reduced the (expensive) hospitalisations while patients believed that their health status has improved. Our study is not a randomised, prospective evaluation and the results may be affected by a number of biases related of selection of patients and patient number is small. Future randomised and perhaps multicenter studies are needed to intensify this conclusion.

\section{Acknowledgements}

This work was supported in part by a CEC project IST1999-13352 with acronym CHS.

\section{References}

[1] Collen MF. Historical evolution of preventive medical informatics in the USA. Meth Inform Med 2000;39:204-7.

[2] Borowitz SM, Wyatt JC. The origin, content and workload of E-mail consultations. JAMA 1998;280:1321-4.

[3] Balas EA, Iakovidis I. Distance technologies for patient monitoring. BMJ 1999;319:1309.

[4] Rosenblatt E. Telephone triage. A common sense approach. RN 2001;64(3):suppl 2-3

[5] Wilson JR, Smith JS, Dahle KL, Ingersoll GL. Impact of home health care on health care costs and hospitalisation frequency in patients with heart failure. Amer J Cardiol 1999;83:615-7.

[6] Cordisco ME, Beniaminovitz A, Hammond K, Mancini D. Use of telemonitoring to decrease the rate of hospitalization in patients with severe congestive heart failure. Amer J Cardiol 1999;84:860-2.

[7] Horowitz JD. Home-based intervention: the next step in treatment of chronic heart failure? Eur Heart J 2000;21:1807-9.

[8] N. Maglaveras, V. Koutkias, I. Chouvarda, D.G. Goulis, A. Avramides, D. Adamidis, G. Louridas, E.A. Balas. Home Care Delivery through the Mobile Telecommunications Platform: The Citizen Health System (CHS) Perspective. International Journal of Medical Informatics 2002;68:99-111.

[9] Albright K, Slater SG. Medical devices in the home: present and future applications. Caring 2000;19(7):36-8.

Address for correspondence.

Nicos Maglaveras. PhD, Associate Professor, Aristotle University, The Medical School, Lab. of Medical Informatics Box 323, 54124 Thessaloniki, GREECE, EMAIL: nicmag@med.auth.gr

\begin{tabular}{|c|c|c|c|c|c|c|c|c|c|c|c|}
\hline \multicolumn{12}{|l|}{ LitVisow | Graph View | } \\
\hline Coniad ad & Conibat thpe & Systole B.P. & $\begin{array}{l}\text { Diascle } \\
\text { B.P. }\end{array}$ & Plose & Weight & Tersper. & Beestless? & $\begin{array}{l}\text { Logs: } \\
\text { monden? }\end{array}$ & Mare tres? & $\begin{array}{l}\text { More } \\
\text { dyppes? }\end{array}$ & Medcation? \\
\hline DWed 24/4/200e 9.33.AM & Calcerier & 127 & 82 & 86 & 81,6 & 35.9 & Yer & No & No & No & Yes \\
\hline Fri26/4/2002954AM & Calcenter & 126 & 78 & 67 & 81.5 & $x$ & Yes & Yes & $Y_{e s}$ & Yes & Yes \\
\hline Man 29/4/2002 11:28 AM & Cal Certer & 121 & 69 & 91 & 82,6 & 36.2 & Yes & Yes & Yes & Yes: & Yes \\
\hline Mon 29/4/2002 400 PM & EOG Trominititi & & & & & & & & & & \\
\hline Wed 1/5/2000 10:07 AM & Calcerier & 116 & 79 & 81 & 83 & 36,4 & Yes & Yes & Yes & Yes & Yes \\
\hline$]_{\mathrm{Fi} i 3 / 20002908 \mathrm{AM}}$ & Cal Certer & 112 & 75 & 83 & 84 & 36,3 & Yes & Yes & Yes & Yes & Yes \\
\hline Wed 8.5/200e 9.30 , AM & Calcerter & 112 & 86 & 102 & 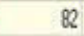 & 36,4 & Yes & No & Yes & Yes & Yes \\
\hline Wed 85/2002 4:CQPM & EOG Termenimi: & & & & & & & & & & \\
\hline
\end{tabular}

Figure 1. Overview of patient monitoring values. Numbers beyond the predetermined range are coloured in red or yellow to attract the physician's attention. 